\title{
Fault Diagnosis of Electric Power Systems Based on Fuzzy Reasoning Spiking Neural P Systems
}

\author{
Tao Wang, Gexiang Zhang, Member, IEEE, Junbo Zhao, Student Member, IEEE, \\ Zhengyou He, Senior Member, IEEE, Jun Wang, and Mario J. Pérez-Jiménez
}

\begin{abstract}
This paper proposes a graphic modeling approach, fault diagnosis method based on fuzzy reasoning spiking neural $P$ systems (FDSNP), for power transmission networks. In FDSNP, fuzzy reasoning spiking neural $P$ systems (FRSN $P$ systems) with trapezoidal fuzzy numbers are used to model candidate faulty sections and an algebraic fuzzy reasoning algorithm is introduced to obtain confidence levels of candidate faulty sections, so as to identify faulty sections. FDSNP offers an intuitive illustration based on a strictly mathematical expression, a good fault-tolerant capacity due to its handling of incomplete and uncertain messages in a parallel manner, a good description for the relationships between protective devices and faults, and an understandable diagnosis model-building process. To test the validity and feasibility of FDSNP, seven cases of a local subsystem in an electrical power system are used. The results of case studies show that FDSNP is effective in diagnosing faults in power transmission networks for single and multiple fault situations with/without incomplete and uncertain SCADA data, and is superior to four methods, reported in the literature, in terms of the correctness of diagnosis results.
\end{abstract}

Index Terms-Electric power system, fault diagnosis, fuzzy production rules, fuzzy reasoning, fuzzy reasoning spiking neural $P$ system, linguistic term, trapezoidal fuzzy number.

\section{INTRODUCTION}

W HEN operating electric power systems (EPS), one of the key objectives is to supply reliable and stable power to customers. Impacted by various factors such as disturbances and equipment failures, interruption of power service or even blackout may happen in power systems [1], [2]. To reduce the loss caused by the two latter undesired events, it is essential for dispatchers to quickly identify the faulty sections in the power system to restore power supply. When a fault occurs, a large number of alarm messages from supervisor control and data acquisition (SCADA) systems are poured into dispatchers' consoles in a short period of time. These messages are often incomplete and uncertain [3]-[6]. Thus, it is necessary to develop

This work was supported by the National Natural Science Foundation of China $(61170016,61373047,61170030)$, the Program for New Century Excellent Talents in Uni-versity (NCET-11-0715) and SWJTU supported project (SWJTU12CX008). Paper no. TPWRS-01113-2013.

T. Wang, G. Zhang, J. Zhao, and Z. He are with the School of Electrical Engineering, Southwest Jiaotong University, Chengdu 610031, China (e-mail: wangatao2005@163.com; zhgxdylan@126.com; junbozhao55589@gmail.com; hezy@swjtu.edu.cn).

J. Wang is with the School of Electrical and Information Engineering, Xihua University, Chengdu 610039, China (e-mail: wangjun@mail.xhu.edu.cn).

M. J. Pérez-Jiménez is with the Research Group on Natural Computing, Department of Computer Science and Artificial Intelligence, University of Sevilla, Sevilla 41012, Spain (e-mail: marper@us.es). a good method to help dispatchers evaluate where the faults are and which sections fail [4].

Fault diagnosis of a power system is a complicated process because it contains lots of sections such as generators, transmission lines, bus bars and transformers which are protected by a protective system consisting of protective relays, circuit breakers (CBs) and communication equipments [5]. In recent decades, fault diagnosis has been implemented by various approaches, such as expert systems (ES) [1], [2], fuzzy logic (FL) [3]-[7], artificial neural networks (ANNs) [8], [9], Petri nets (PNs) [4], [5], [10], Bayesian networks (BNs) [11], [12], multiagent systems (MAS) [13], [14], optimization methods (OM) [15]-[17], cause-effect networks (CE-Nets) [6], [7], [18], and information theory (IT) [19], [20]. Each method has its own merits and demerits.

ES is the earliest artificial intelligence method for power system fault diagnosis. ES makes full use of experts' knowledge, but it has a slow inference speed due to its sequential search nature, and the difficulties of designing and maintaining a rule-based knowledge system [2], [18]. FL expresses imprecision and uncertainty, but it needs to be combined with other methods [18], [20]. ANNs have the advantages of good tolerance and strong learning ability, and the disadvantages of the empirical design of network structures and parameters, premature convergence and the need for numerous samples [18]. The major features of PN-based methods are graphical knowledge representation and parallel information processing, however, bad tolerance and combinatorial explosion in large power networks are their weaknesses [6]. Fault diagnosis models based on BNs are intuitive and can find relationships of causality between data, but it is difficult for these methods to obtain accurate prior probabilities and model complex power grid [12], [20]. In MAS, several agents corresponding to several methods cooperate to fulfill fault diagnosis, but how to properly combine different methods and how these agents cooperate are unsolved issues [13], [20]. Although OM may obtain globally optimal solutions of a complex fault diagnosis problem, it is not easy to construct an objective function reflecting the discrepancy between the expected and actual states of protective devices and to adjust the parameters in the optimization model [16], [18]. CE-Nets are a graphical tool for knowledge representation with easy algebraic reasoning and parallel information processing ability, but their fault tolerance needs to be improved and their forward reasoning strategy makes CE-Nets unable to visually represent all possible combinations of main, first and second protections [18], [21]. IT-based methods are a novel approach emerging with the informatization of power systems. To a certain extent, these methods deal with the uncertainty in 
failure processes with fast diagnosis speed, but it is difficult to dynamically describe the fault information needed [20]. Therefore, much attention should be paid to the improvements of the aforementioned methods and the exploration of new ones to solve fault diagnosis problems.

This paper discusses a novel fault diagnosis approach for power systems by using membrane systems or P systems, which are theoretical computing devices in the area of membrane computing. As a newly attractive research field of computer science, membrane computing, formally introduced by Păun [22], aims at abstracting computing models from the structure and the functioning of living cells, as well as from the way that cells are organized in tissues or higher order structures. A spiking neural P system (SN P system) is the type of P system inspired by the neurophysiological behavior of neurons sending electrical impulses (spikes) along axons from presynaptic neurons to postsynaptic neurons in a distributed and parallel manner [23]. An SN $\mathrm{P}$ system can be considered as a set of nodes representing neurons in a directed graph whose arcs express synaptic connections among neurons. The contents of each neuron are composed of several copies of a single object type. Likewise, each neuron has a finite set of firing (spiking) and forgetting rules. Firing rules send information between neurons in the form of spikes and forgetting rules remove spikes from neurons. The rules associated with each neuron are used in a sequential manner, but neurons communicate with each other in parallel. Recently, SN P systems have become a hot topic in membrane computing [24]-[30].

SN P systems are a class of distributed and parallel computing models with good understandability and dynamics [28], [29]. The fault occurrence in power systems is a discrete and dynamical process [4]-[6]. Thus, SN P systems can be used for diagnosing faults in power systems. In the preliminary work, we discussed the knowledge representation ability of FRSN P systems [26], [27]. To successfully fulfill diagnosis knowledge representation and reasoning, an FRSN P system with real numbers was presented in [28], where only one transformer was considered. In these systems, the potential value that a spike can take within a neuron and the truth value of a fuzzy proposition (or confidence factor of a production rule) are represented by real numbers in $[0,1]$. In [21], an approach based on the FRSN P system, presented in [28], for fault diagnosis of power systems was discussed and three different applications were used to verify its effectiveness. How to handle the incompleteness and uncertainty of the alarm information in different power systems is worth further discussing.

To handle incompleteness and uncertainty in power transmission network fault diagnosis in electric power systems, a method based on FRSN P systems, FDSNP, is developed in this paper. In FDSNP, an FRSN P system with trapezoidal fuzzy numbers is introduced to model candidate faulty sections and an algebraic fuzzy reasoning algorithm of the FRSN P system is presented for matrix-based fault reasoning to obtain confidence levels of candidate faulty sections and identify faulty sections. In order to make the process of building diagnosis models based on FRSN $P$ systems easily understandable, fault fuzzy production rule sets to obtain the relationships between protective devices and faults are presented.

The main contribution of this work is summarized as follows. This study provides a fault diagnosis method based on FRSN

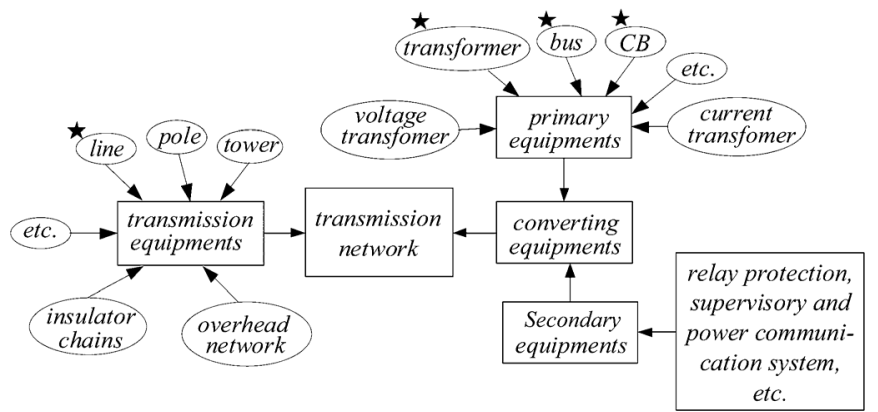

Fig. 1. Equipments and sections in a power transmission network.

P systems with trapezoidal fuzzy numbers for power transmission networks. The main ideas of this method include the introduction of trapezoidal fuzzy numbers into FRSN P systems, the fuzzy reasoning algorithm within the framework of FRSN P systems with trapezoidal fuzzy numbers and the fault fuzzy production rule sets based on syntactical ingredients of FRSN $P$ systems with trapezoidal fuzzy numbers. The use of trapezoidal fuzzy numbers is helpful to express potential values of spikes contained in neurons and the fuzzy truth values of the neurons and consequently allows us to handle incompleteness and uncertainty in power systems. The presented reasoning algorithm uses an easily understandable description and has various kinds of synaptic matrices to describe relationships among faulty sections, protective relays and their CBs more flexible and effective. The fault fuzzy production rule sets for main sections including transmission lines, buses and transformers in transmission networks can offer the causality between the faults and the statuses of protective relays and their corresponding CBs in an intuitive and visual way.

The remainder of this paper is organized as follows. Section II states the problem to solve. Section III presents FDSNP. Following, seven case studies are provided in Section IV. Conclusions are finally drawn in Section V.

\section{Problem Description}

Strictly speaking, fault diagnosis includes fault detection, fault section identification, fault type estimation, failure isolation and recovery [17]. Among the five processes, fault section identification is especially important [4], [5]. A power transmission network is composed of transmission equipments and converting equipments, as each of which consists of many kinds of sections as shown in Fig. 1. In this paper, we focus on the fault diagnosis of a power transmission network because it is one of the major networks in power systems. In this study, the faults of lines, buses and transformers in power transmission networks are diagnosed by using the statuses of protective relays and circuit breakers (CBs) because they are normally read from a power SCADA system. The protective relays consist of main protective relays (MPRs), first backup protective relays (FBPRs) and second backup protective relays (SBPRs). It is worth pointing out that there is not any FBPR for buses. Fig. 2 shows a schematic illustration of the transmission network with sections and protective relays considered in this study.

Protective relays of transmission lines are of two types: sending end protective relays and receiving end protective relays. To illustrate the operational rules of different 


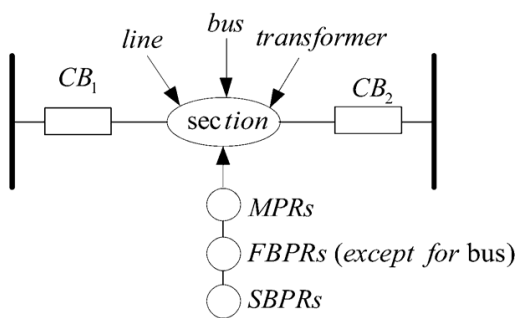

Fig. 2. Schematic illustration of the power transmission network with sections and protective relays considered in this study.

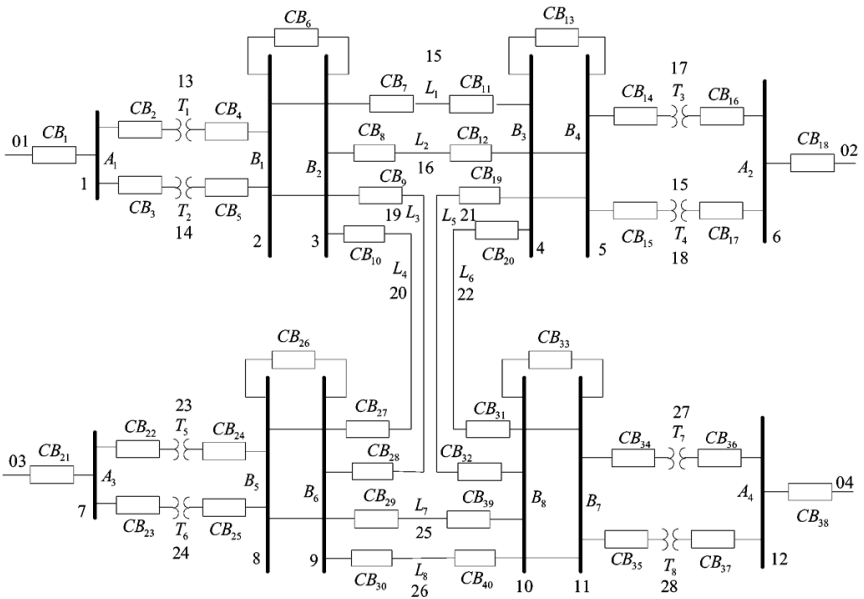

Fig. 3. Local sketch map of the protection system of an EPS.

types of protections, a local sketch map of the protection system of an EPS is chosen from [4], [17] and shown in Fig. 3, which includes 28 system sections, $40 \mathrm{CBs}$ and 84 protective relays. For the convenience of description, some notations are described as follows. A single bus, double bus, transformer and line are represented by $A, B, T$, and $L$, respectively. $S$ and $R$ represent the sending and receiving ends of the line $L$, respectively. $m, p$, and $s$ denote the main protection, the first backup protection and the second backup protection, respectively. The 28 sections $\left(S_{1} \sim S_{28}\right)$ are labeled as $A_{1}, \ldots$, $A_{4}, T_{1}, \ldots, T_{8}, B_{1}, \ldots, B_{8}, L_{1}, \ldots, L_{8}$ and the $40 \mathrm{CBs}$ $\left(C_{1} \sim C_{40}\right)$ are labeled as $C B_{1}, C B_{2}, \ldots, C B_{40}$. The 84 protective relays are composed of 36 main ones, $r_{1} \sim r_{36}$ represented by $A_{1 m}, \ldots, A_{4 m}, T_{1 m}, \ldots, T_{8 m}, B_{1 m}, \ldots, B_{8 m}, L_{1 S m}, \ldots$, $L_{8 S m}, L_{1 R m}, \ldots, L_{8 R m}$, and $r_{37} \sim r_{84}$ denoted as $T_{1 p}, \ldots$, $T_{8 P}, T_{1 s}, \ldots, T_{8 s}, L_{1 S p}, \ldots, L_{8 S p}, L_{1 R p}, \ldots, L_{8 R p}, L_{1 S s}, \ldots$, $L_{8 S s}, L_{1 R s}, \ldots, L_{8 R s}$.

The operational rules of the protective relays for the three kinds of sections, lines, buses and transformers, are described in [17] as follows.

1) Protective relays of lines

Both ends of a line have their own main, first and second protections. When the main protective relays of a line operate, $\mathrm{CBs}$ connected to the line are tripped. For example, if line $L_{7}$ fails, MPRs $L_{7 S m}$ and $L_{7 R m}$ are operate to trip $C B_{29}$ and $C B_{39}$, respectively. Likewise, when the main protections of a line fail to operate, the first backup protective relays operate to trip CBs connected to the line. For example, if line $L_{7}$ fails and main protection relay (MPR) $L_{7 S m}$ fails to operate, first backup protective relay (FBPR)

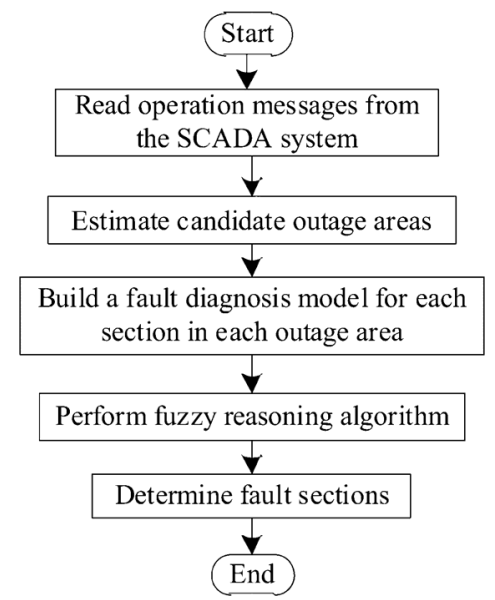

Fig. 4. Flowchart of FDSNP.

$L_{7 S p}$ operates to trip $C B_{29}$. If line $L_{7}$ fails and MPR $L_{7 R m}$ fails to operate, FBPR $L_{7 R p}$ operates to trip $C B_{39}$. When the adjacent regions of a line fail and their protections fail to operate, the second backup protections operate to protect the line. For example, if section $B_{8}$ fails and $C B_{39}$ fails to trip off, second backup protective relay (SBPR) $L_{7 S s}$ operates to trip $C B_{29}$. If section $B_{5}$ fails and $C B_{29}$ fails to trip off, SBPR $L_{7 R s}$ operates to trip $C B_{39}$.

2) Protective relays of buses

When the main protective relays of a bus operate, all CBs direct connected to the bus will be tripped. For example, if bus $A_{1}$ fails, MPR $A_{1 m}$ operates to trip $C B_{1}, C B_{2}$, and $C B_{3}$. Similarly, if bus $B_{8}$ fails, MPR $B_{8 m}$ operates to trip $C B_{32}, C B_{33}$, and $C B_{39}$.

3) Protective relays of transformers

When the main protective relays of a transformer operate, all CBs connected to the transformer are tripped. For example, if transformer $T_{3}$ fails, MPR $T_{3 m}$ operates to trip $C B_{14}$ and $C B_{16}$. Likewise, when the main protections of a transformer fail to operate, the first backup protective relays operates to trip CBs connected to the transformer. For example, if transformer $T_{3}$ fails and MPR $T_{3 m}$ fails to operate, FBPR $T_{3 p}$ operates to trip $C B_{14}$ and $C B_{16}$. When the adjacent regions of the transformer fail and their protections fail to operate, the second backup protections operate to protect the transformer. For example, if bus $A_{2}$ fails and $C B_{16}$ fails to trip off, SBPR $T_{3 s}$ operates to trip $C B_{16}$ to protect $T_{3}$.

In this study, FDSNP is used to fulfill fault diagnosis of main sections, transmission lines, buses and transformers, in power transmission networks when some incomplete and uncertain status information about protective relays and CBs is detected. The flowchart of FDSNP is shown in Fig. 4. First, the status information is read from the SCADA system. Second, outage areas are identified to obtain the suspected fault sections using network topology analysis method [10], [31]. Third, a fault diagnosis model for each section in each candidate outage area is built. Then, each fault diagnosis model performs a fuzzy reasoning algorithm to obtain fault confidence levels of suspicious fault sections. Finally, faulty sections are determined according to their fault confidence levels. The key ingredients and steps of FDSNP are presented in detail in Section III. 


\section{FDSNP}

This section presents a graphic modeling approach, FDSNP, for fault diagnosis of power transmission networks based on FRSN P systems with trapezoidal fuzzy numbers. We first give the definition of an FRSN P system with trapezoidal fuzzy numbers and then describe its algebraic fuzzy reasoning algorithm. Subsequently, the fault fuzzy production rule sets for main sections including transmission lines, buses and transformers in power transmission networks are presented. Finally, FDSNP is algorithmically illustrated.

\section{A. FRSN P Systems With Trapezoidal Fuzzy Numbers}

Definition 1: An FRSN P system with trapezoidal fuzzy numbers (with degree $m \geq 1$ ) is a construct

$$
\Pi=\left(O, \sigma_{1}, \ldots, \sigma_{m}, \text { syn }, \text { in }, \text { out }\right)
$$

where

1) $O=\{a\}$ is a singleton alphabet ( $a$ is called spike);

2) $\sigma_{1}, \ldots, \sigma_{m}$ are neurons of the form $\sigma_{i}=\left(\theta_{i}, c_{i}, r_{i}\right), 1 \leq$ $i \leq m$, where

a) $\theta_{i}$ is a trapezoidal fuzzy number in $[0,1]$ representing the potential value of spikes (i.e., the value of electrical impulses) contained in neuron $\sigma_{i}$;

b) $c_{i}$ is a trapezoidal fuzzy number in $[0,1]$ representing the fuzzy truth value corresponding to neuron $\sigma_{i}$;

c) $r_{i}$ represents a firing (spiking) rule associated with neuron $\sigma_{i}$ of the form $E / a^{\theta} \rightarrow a^{\beta}$, where $E$ is a regular expression, and $\theta$ and $\beta$ are trapezoidal fuzzy numbers in $[0,1]$.

3) syn $\subseteq\{1,2, \ldots, m\} \times\{1,2, \ldots, m\}$ with $i \neq j$ for all $(i, j) \in$ syn, $1 \leq i, j \leq m$, is a directed graph of synapses between the linked neurons;

4) in, out $\in\{1,2, \ldots, m\}$ indicate the input neuron set and the output neuron set of $\Pi$, respectively.

In an FRSN $\mathrm{P}$ system, the pulse value contained in each neuron is not the number of spikes represented by a real number, but a trapezoidal fuzzy number in $[0,1]$, which can be interpreted as the potential value of spikes contained in neuron $\sigma_{i}$. The motivation for the introduction of trapezoidal fuzzy numbers comes from three aspects. First of all, due to experts' subjectivity, professional knowledge acquisition has various uncertainties. Subsequently, human knowledge in the real world, such as knowledge in fault diagnosis process, is usually expressed by using linguistic terms with a certain degree of uncertainty. For example, we often use fuzzy concepts (absolutely-false, very-low, low, medium-low, medium, medium-high, high, very-high, absolutely-high) to describe a degree of uncertainty. In addition, the knowledge in practical applications may contain a certain degree of uncertainty. For instance, the operation process of protective devices in fault diagnosis usually includes uncertainly protective messages such as maloperation and misinformation.

The trapezoidal fuzzy number in Definition 1 can be parameterized by a 4-tuple $\tilde{A}=\left(a_{1}, a_{2}, a_{3}, a_{4}\right)$, as shown in Fig. 5, where $a_{1}, a_{2}, a_{3}$ and $a_{4}$ are real numbers such that $a_{1}<a_{2}<$

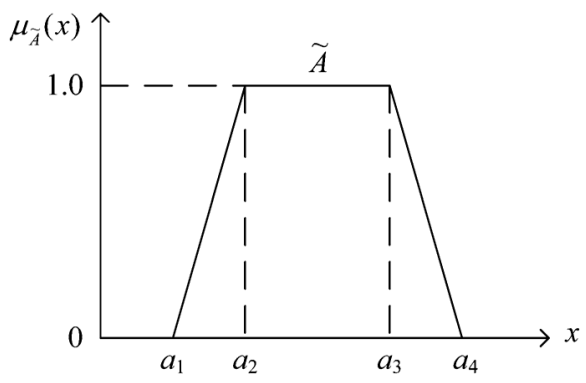

Fig. 5. Trapezoidal fuzzy number.

$a_{3}<a_{4}$, which are the four horizontal axis values of the trapezoid. The membership function $\mu_{\tilde{A}}(x)$ of the trapezoidal fuzzy number $\tilde{A}$ is defined as follows:

$$
\mu_{\tilde{A}}(x)= \begin{cases}0, & x \leq a_{1} \\ \frac{x-a_{1}}{a_{2}-a_{1}}, & a_{1}<x \leq a_{2} \\ 1, & a_{2}<x \leq a_{3} \\ \frac{a_{4}-x}{a_{4}-a_{3}}, & a_{3}<x \leq a_{4} \\ 0, & x>a_{4} .\end{cases}
$$

Let $\tilde{A}$ and $\tilde{B}$ be two trapezoidal fuzzy numbers, $\tilde{A}=\left(a_{1}, a_{2}, a_{3}, a_{4}\right)$ and $\tilde{B}=\left(b_{1}, b_{2}, b_{3}, b_{4}\right)$. The arithmetic operations of the trapezoidal fuzzy numbers $\tilde{A}$ and $\tilde{B}$ are listed as follows. More operations can be seen in [6] and [32].

1) Addition $\oplus: \tilde{A} \oplus \tilde{B}=\left(a_{1}, a_{2}, a_{3}, a_{4}\right) \oplus\left(b_{1}, b_{2}, b_{3}, b_{4}\right)=$ $\left(a_{1}+b_{1}, a_{2}+b_{2}, a_{3}+b_{3}, a_{4}+b_{4}\right)$;

2) Multiplication $\otimes: \tilde{A} \otimes \tilde{B}=\left(a_{1}, a_{2}, a_{3}, a_{4}\right) \otimes$ $\left(b_{1}, b_{2}, b_{3}, b_{4}\right)=\left(a_{1} \times b_{1}, a_{2} \times b_{2}, a_{3} \times b_{3}, a_{4} \times b_{4}\right)$.

We define four logic operations as follows, where $A$ and $B$ are trapezoidal fuzzy numbers, and $a, b$ are real numbers.

1) Minimum operator $\wedge: a \wedge b=\min (a, b)$;

2) Maximum operator $\vee: a \vee b=\max (a, b)$;

3) and $\left(A: A \bowtie B=\left(a_{1}, a_{2}, a_{3}, a_{4}\right) \oplus\left(b_{1}, b_{2}, b_{3}, b_{4}\right)=\right.$ $\left(\left(a_{1} \wedge b_{1}\right),\left(a_{2} \wedge b_{2}\right),\left(a_{3} \wedge b_{3}\right),\left(a_{4} \wedge b_{4}\right)\right)$

4) or $\nabla: A \oslash B=\left(a_{1}, a_{2}, a_{3}, a_{4}\right) \emptyset\left(b_{1}, b_{2}, b_{3}, b_{4}\right)=\left(\left(a_{1} \vee\right.\right.$ $\left.\left.b_{1}\right),\left(a_{2} \vee b_{2}\right),\left(a_{3} \vee b_{3}\right),\left(a_{4} \vee b_{4}\right)\right)$.

We also define a scalar multiplication operation as follows, where $A$ is a trapezoidal fuzzy number and $b$ is a real number.

1) Scalar Multiplication: $b A=b\left(a_{1}, a_{2}, a_{3}, a_{4}\right)=$ $\left(b a_{1}, b a_{2}, b a_{3}, b a_{4}\right)$.

With regard to the pulse value $\theta_{i}$, if $\theta_{i}>(0,0,0,0)$, neuron $\sigma_{i}$ contains a spike with value $\theta_{i}$, otherwise, the neuron contains no spike. The firing condition $E=a^{n}$ means that the spiking rule associated with neuron $\sigma_{i}$ can be applied if and only if the number of spikes that neuron $\sigma_{i}$ receives at any computational step equals $n$, otherwise, the firing rule cannot be applied. If the number of spikes that neuron $\sigma_{i}$ receives is less than $n$, neuron $\sigma_{i}$ performs the operation $($ or $\varnothing$ on the potential values carried by these spikes to update its pulse value.

According to their usage, in an FRSN P system the neurons are divided into two categories: proposition neurons and rule neurons. Each neuron $\sigma_{i}$ corresponds to either a proposition or a fuzzy production rule, which will be described later in this section. Thus, the trapezoidal fuzzy number $c_{i}$ can be understood as either the fuzzy truth value of a proposition or the certainty factor of a fuzzy production rule. 
Definition 2: A proposition neuron, as shown in Fig. 6, corresponds to a proposition in the fuzzy production rules. Such a neuron is represented by the symbol $P$.

The fuzzy truth value of a proposition neuron equals to the fuzzy truth value of the proposition corresponding to this neuron. If such a proposition neuron receives one spike, i.e., $n=1$, it will fire and emit a spike. The parameter $\beta$ of the firing rule contained in such a proposition neuron is identical to $\theta$. If a proposition neuron is an input, then its pulse value $\theta$ equals to the fuzzy truth value $c$ of this neuron. Otherwise, if there is only one presynaptic rule neuron, then $\theta$ equals to the pulse value transmitted from this neuron. In any other case, $\theta$ equals to the result of the operation $(\mathbb{A})$ on all pulse values received from its presynaptic rule neurons.

There are three types of rule neurons: general, and and or, which are represented by the three symbols $\ominus, \otimes$ and $\emptyset$, respectively. We use $R$ to denote a rule neuron. If the number of spikes a rule neuron receives equals the number of its presynapses, it will fire and emit a spike. In what follows we define each type of rule neurons.

Definition 3: A general rule neuron $\ominus$, as shown in Fig. 7 (i), corresponds to a fuzzy production rule which has only one proposition in the antecedent part of the rule. The consequent part of the fuzzy production rule may contain one or more propositions.

A general rule neuron has only one presynaptic proposition neuron and one or more postsynaptic proposition neurons. The fuzzy truth value of a general rule neuron equals to the certainty factor of the fuzzy production rule corresponding to its neuron. If a general rule neuron receives a spike with potential value $\theta$ and its firing condition is satisfied, then the neuron fires and produces a new spike with potential value $\beta=\theta \otimes c$.

Definition 4: An and rule neuron $\bigotimes$, as shown in Fig. 7 (ii), corresponds to the fuzzy production rule which has more than one proposition with an and relationship in the antecedent part of the rule. The consequent part of the fuzzy production rule contains only one proposition.

An and rule neuron has more than one presynaptic proposition neuron and only one postsynaptic proposition neuron. The fuzzy truth value of an and rule neuron equals to the certainty factor of the fuzzy production rule corresponding to its neuron. If an and rule neuron receives $k$ spikes with potential values $\theta_{1}, \theta_{2}, \ldots, \theta_{k}$, respectively, and its firing condition is satisfied, then the neuron fires and produces a new spike with the potential value $\beta=\left(\theta_{1} \otimes \ldots \otimes \theta_{k}\right) \otimes c$.

Definition 5: An or rule neuron $\bigotimes$, as shown in Fig. 7 (iii), corresponds to the fuzzy production rule which has more than one proposition with an or relationship in the antecedent part of the rule. The consequent part of the fuzzy production rule contains only one proposition.

An or rule neuron has more than one presynaptic proposition neurons and only one postsynaptic proposition neuron. The fuzzy truth value of an or rule neuron equals to the certainty factor of the fuzzy production rule corresponding to its neuron. If an or rule neuron receives $k$ spikes with potential values $\theta_{1}, \theta_{2}, \ldots, \theta_{k}$, respectively, and its firing condition is satisfied, then the neuron fires and produces a new spike with the potential value $\beta=\left(\theta_{1} \otimes \ldots \otimes \theta_{k}\right) \otimes c$.

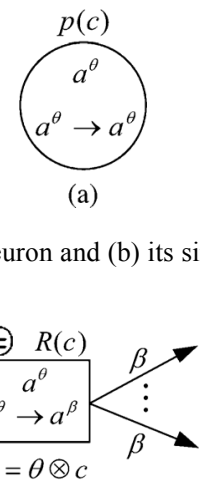

(a)

(ii)

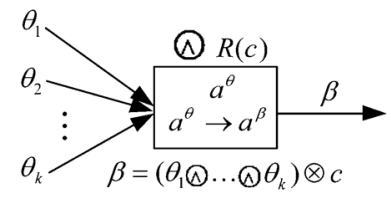

(a)

(iii)

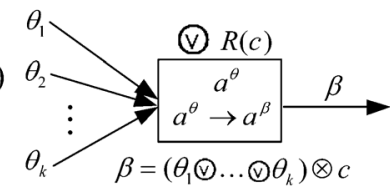

(a)

(i)

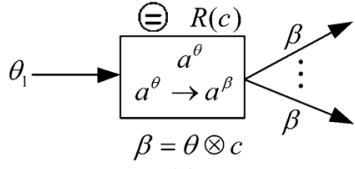

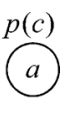

(b)

Fig. 6. (a) Proposition neuron and (b) its simplified form.

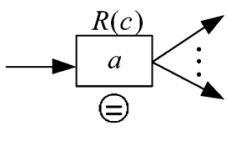

(b)

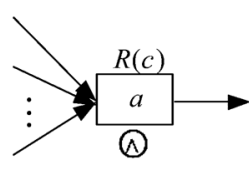

(b)
Fig. 7. Rule neurons. (i) A general rule neuron (a) and its simplified form (b); (ii) An and rule neuron (a) and its simplified form (b); (iii) An or rule neuron (a) and its simplified form (b).$$
\text { (a) } \sigma_{j} \bigodot_{p_{j}\left(\theta_{j}\right)}^{a} \longrightarrow \underbrace{R_{i}\left(c_{i}\right)}_{\sigma_{l} \Theta} \longrightarrow \bigodot_{p_{k}\left(\theta_{k}\right)} \sigma_{k}
$$
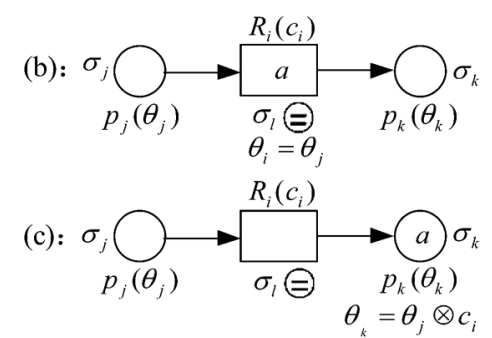

Fig. 8. Modeling process of Type 1 using one FRSN P system.

In what follows, we use FRSN P systems to model these fuzzy production rules, which will be used to model fault diagnosis in power systems. We consider four types of fuzzy production rules. An FRSN P system is used to model one or more fuzzy production rules. In the following description, $R_{i}\left(i=1, \ldots, N_{r}\right)$ is the $i$ th fuzzy production rule, $N_{r}$ represents the number of fuzzy production rules, $c_{i}$ is a trapezoidal fuzzy number in $[0,1]$ representing the certainty factor of $R_{i}, p_{j}\left(1 \leq j \leq N_{p}\right)$ is the $j$ th proposition appearing in the antecedent or consequent part of $R_{i}, N_{p}$ represents the number of proportions, and $\theta_{j}$ is a trapezoidal fuzzy number in $[0,1]$ representing the fuzzy truth value of proposition $p_{j}$.

Type 1: $R_{i}\left(c_{i}\right): p_{j}\left(\theta_{j}\right) \rightarrow p_{k}\left(\theta_{k}\right)\left(1 \leq j, k \leq N_{p}\right)$. The modeling process of this rule type by using one FRSN P system is shown in Fig. 8, where (a), (b), and (c) represent spike $a$ being transmitted from input neuron $\sigma_{j}$ to output neuron $\sigma_{k}$. The fuzzy truth value of the proposition $p_{k}$ is $\theta_{k}=\theta_{j} \otimes c_{i}$. 
(a):

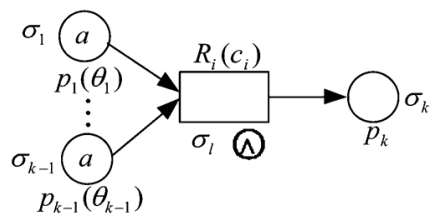

(b):

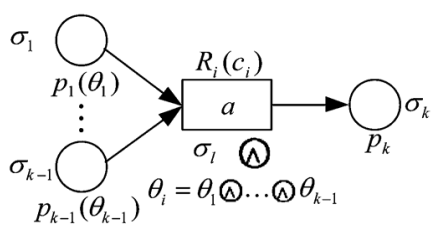

(c)

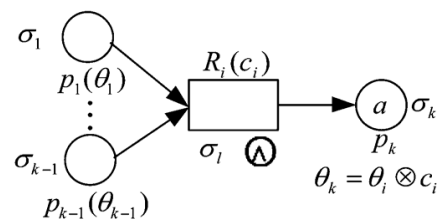

Fig. 9. Modeling process of Type 2 using one FRSN P system.

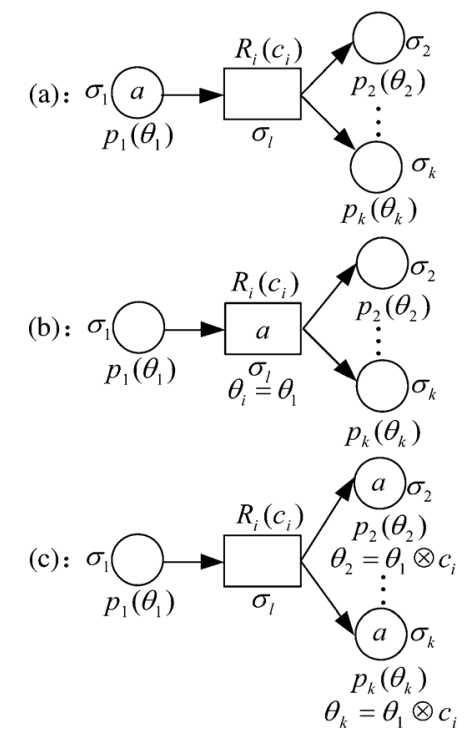

Fig. 10. Modeling process of Type 3 using one FRSN P system.

Type 2: $R_{i}\left(c_{i}\right): p_{1}\left(\theta_{1}\right) \bigotimes \ldots\left(p_{k-1}\left(\theta_{k-1}\right) \rightarrow p_{k}\left(\theta_{k}\right)\right.$. The process of this rule type modeled by using one FRSN $P$ system is shown in Fig. 9, where (a), (b), and (c) represent spike $a$ being transmitted from input neurons $\sigma_{1}, \sigma_{2}, \ldots, \sigma_{k-1}$ to output neuron $\sigma_{k}$. The fuzzy truth value of the proposition $p_{k}$ is $\theta_{k}=\left(\theta_{1} \otimes \ldots \otimes \theta_{k-1}\right) \otimes c_{i}$.

Type 3: $R_{i}\left(c_{i}\right): p_{1}\left(\theta_{1}\right) \rightarrow p_{2}\left(\theta_{2}\right) \otimes \ldots \otimes p_{k}\left(\theta_{k}\right)$. The process of this rule type modeled by using one FRSN P system is shown in Fig. 10, where (a), (b), and (c) represent spike $a$ being transmitted from input neuron $\sigma_{1}$ to output neurons $\sigma_{2}, \sigma_{3}, \ldots, \sigma_{k}$. The fuzzy truth values of the propositions $p_{2}, p_{3}, \ldots, p_{k}$ are identical, i.e., $\theta_{2}=\theta_{3}=\ldots=\theta_{k}=\theta_{1} \otimes c_{i}$.

Type 4: $R_{i}\left(c_{i}\right): p_{1}\left(\theta_{1}\right) \bigotimes \ldots \bigotimes p_{k-1}\left(\theta_{k-1}\right) \rightarrow p_{k}\left(\theta_{k}\right)$. The process of this rule type modeled by using one FRSN P system is shown in Fig. 11, where (a), (b), and (c) represent spike $a$ being transmitted from input neurons $\sigma_{1}, \sigma_{2}, \ldots, \sigma_{k-1}$ into output neuron $\sigma_{k}$. The fuzzy truth value of the proposition $p_{k}$ is $\theta_{k}=\left(\theta_{1} \oslash \ldots \otimes \theta_{k-1}\right) \otimes c_{i}$.

The fuzzy truth values of the propositions appearing in the fuzzy production rules and the certainty factor of each fuzzy production rule can also be described by using linguistic terms, (a):

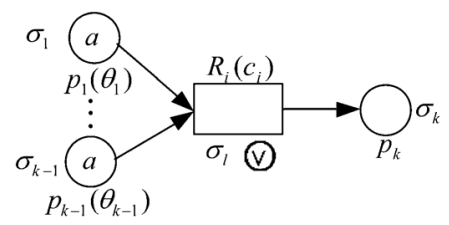

(b):

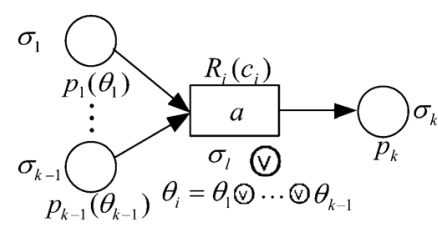

(c):

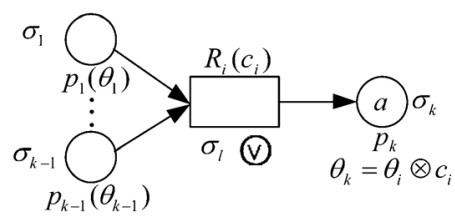

Fig. 11. Modeling process of Type 4 using one FRSN P system.

TABLE I

LINGUISTIC TERMS AND THEIR CORRESPONDING TRAPEZOIDAL FUZZY NUMBERS

\begin{tabular}{ll}
\hline Linguistic Terms & Trapezoidal Fuzzy Numbers \\
\hline absolutely-false (AF) & $(0,0,0,0)$ \\
very-low (VL) & $(0,0,0.02,0.07)$ \\
low (L) & $(0.04,0.1,0.18,0.23)$ \\
medium-low (ML) & $(0.17,0.22,0.36,0.42)$ \\
medium (M) & $(0.32,0.41,0.58,0.65)$ \\
medium-high (MH) & $(0.58,0.63,0.80,0.86)$ \\
high (H) & $(0.72,0.78,0.92,0.97)$ \\
very-high (VH) & $(0.975,0.98,1,1)$ \\
absolutely-high (AH) & $(1,1,1,1)$ \\
\hline
\end{tabular}

which are represented by the trapezoidal fuzzy numbers shown in Table I.

\section{B. Fuzzy Reasoning Algorithm}

This subsection presents an algebraic fuzzy reasoning algorithm for FRSN P systems with trapezoidal fuzzy numbers. If the fuzzy truth values of the propositions corresponding to the input proposition neurons are given, the algorithm reasons out the fuzzy truth values of other unknown propositions corresponding to the other proposition neurons in the FRSN P system. The reasoning results are the fuzzy truth values of the propositions corresponding to the output neurons.

Let us assume that the FRSN P system used for knowledge representation and reasoning contains $s$ proposition neurons and $t$ rule neurons, each of which may be general, and or or rule neurons. Thus, the total number of neurons is $m=s+t$. To explain this reasoning algorithm, we first introduce some vectors and matrices as follows.

1) $\boldsymbol{\theta}=\left(\theta_{1}, \theta_{2}, \ldots, \theta_{s}\right)^{T}$ is a vector containing the fuzzy truth values of the $s$ proposition neurons, where $\theta_{i}$ is a trapezoidal fuzzy number in $[0,1]$ representing the pulse value contained in the $i$ th proposition neuron, $1 \leq i \leq s$. If there is not any spike contained in a proposition neuron, its pulse value is "unknown" or $(0,0,0,0)$.

2) $\boldsymbol{\delta}=\left(\delta_{1}, \delta_{2}, \ldots, \delta_{t}\right)^{T}$ is a vector containing the fuzzy truth values of the $t$ rule neurons, where $\delta_{j}$ is a trapezoidal fuzzy number $[0,1]$ representing the pulse value contained in 
the $j$ th rule neuron, $1 \leq j \leq t$. If there is not any spike contained in a rule neuron, its pulse value is "unknown" or $(0,0,0,0)$.

3) $\boldsymbol{C}=\operatorname{diag}\left(c_{1}, c_{2}, \ldots, c_{t}\right)$ is a diagonal matrix, where $c_{j}$ $(1 \leq j \leq t)$ is a trapezoidal fuzzy number representing the certainty factor of the $j$ th fuzzy production rule.

4) $\boldsymbol{D}_{1}=\left(d_{i j}\right)_{s \times t}$ is a synaptic matrix representing the directed connection from proposition neurons to general rule neurons. If there is a directed arc (synapse) from the proposition neuron $\sigma_{i}$ to the general rule neuron $\sigma_{j}, d_{i j}=1$, otherwise, $d_{i j}=0$.

5) $\boldsymbol{D}_{2}=\left(d_{i j}\right)_{s \times t}$ is a synaptic matrix representing the directed connection from proposition neurons to and rule neurons. If there is a directed arc (synapse) from the proposition neuron $\sigma_{i}$ to the and rule neuron $\sigma_{j}, d_{i j}=1$, otherwise, $d_{i j}=0$.

6) $\boldsymbol{D}_{3}=\left(d_{i j}\right)_{s \times t}$ is a synaptic matrix representing the directed connection from proposition neurons to or rule neurons. If there is a directed arc (synapse) from the proposition neuron $\sigma_{i}$ to the $o r$ rule neuron $\sigma_{j}, d_{i j}=1$, otherwise, $d_{i j}=0$.

7) $\boldsymbol{E}=\left(e_{j i}\right)_{t \times s}$ is a synaptic matrix representing the directed connection from rule neurons to proposition neurons. If there is a directed arc (synapse) from the rule neuron $\sigma_{j}$ to the proposition neuron $\sigma_{i}, e_{j i}=1$, otherwise, $e_{j i}=0$.

Subsequently, we introduce following three multiplication operations.

1) $\odot: \boldsymbol{C} \odot \boldsymbol{\delta}=\left(c_{1} \otimes \delta_{1}, c_{2} \otimes \delta_{2}, \ldots, c_{t} \otimes \delta_{t}\right)^{T}$. Likewise, $\boldsymbol{D}^{T} \odot \boldsymbol{\theta}=\left(\bar{d}_{1}, \bar{d}_{2}, \ldots, \bar{d}_{t}\right)^{T}$, where $\bar{d}_{j}=d_{1 j} \theta_{1} \oplus d_{2 j} \theta_{2} \oplus$ $\ldots \oplus d_{s j} \theta_{s}, j=1,2, \ldots, t$.

2) $\odot: \boldsymbol{D}^{T} \odot \boldsymbol{\theta}=\left(\bar{d}_{1}, \bar{d}_{2}, \ldots, \bar{d}_{t}\right)^{T}$, where $\bar{d}_{j}=$ $d_{1 j} \theta_{1} ه d_{2 j} \theta_{2} \bowtie \ldots \bowtie d_{s j} \theta_{s}, j=1,2, \ldots, t$.

$3) \circledast: \quad \boldsymbol{E}^{T} \circledast \boldsymbol{\delta}=\left(\overline{e_{1}}, \overline{e_{2}}, \ldots, \overline{e_{s}}\right)^{T}$, where $\overline{e_{i}}=$ $e_{1 i} \delta_{1} \oslash e_{2 i} \delta_{2} \oslash \ldots \nabla e_{t i} \delta_{t}, i=1,2, \ldots, s$.

What follows is a description of a fuzzy reasoning algorithm for an FRSN P system.

Input: the fuzzy truth values of the propositions corresponding to the input proposition neurons

Output: the fuzzy truth values of the propositions corresponding to the output proposition neurons

Step 1) Let $g=0$ be the reasoning step;

Step 2) Set initial values of $\boldsymbol{D}_{1}, \boldsymbol{D}_{2}, \boldsymbol{D}_{3}, \boldsymbol{E}, \boldsymbol{C}$ and the termination condition $\mathbf{0}_{1}=$ (unknown, unknown, ... unknown $)_{t}^{T}$. The initial values of $\boldsymbol{\theta}$ and $\boldsymbol{\delta}$ are set to $\boldsymbol{\theta}_{g}=$ $\left(\theta_{1 g}, \theta_{2 g}, \ldots, \theta_{s g}\right)$ and $\boldsymbol{\delta}_{g}=\left(\delta_{1 g}, \delta_{2 g}, \ldots, \delta_{t g}\right)$, respectively.

Step 3) $g$ is increased by one.

Step 4) The firing condition of each input neuron $(g=1)$ or each proposition neuron $(g>1)$ is evaluated. If the condition is satisfied and there is a presynaptic rule neuron, the neuron fires and transmits a spike to the next rule neuron.

Step 5) Compute the fuzzy truth value vector $\boldsymbol{\delta}_{g}$ according to $(2)$ :

$$
\boldsymbol{\delta}_{g}=\left(\boldsymbol{D}_{1}^{T} \odot \boldsymbol{\theta}_{g-1}\right) \oplus\left(\boldsymbol{D}_{2}^{T} \odot \boldsymbol{\theta}_{g-1}\right) \oplus\left(\boldsymbol{D}_{3}^{T} \circledast \boldsymbol{\theta}_{g-1}\right) .
$$

Step 6) If $\boldsymbol{\delta}_{g}=\mathbf{0}_{1}$, the algorithm stops and outputs the reasoning results, otherwise, it go to Step 7).

Step 7) Evaluate the firing condition of each rule neuron. If the condition is satisfied, the rule neuron fires and transmits a spike to the next proposition neuron.

Step 8) Compute the fuzzy truth value vector $\boldsymbol{\theta}_{g}$ according to (3). Go to Step 3):

$$
\theta_{g}=\boldsymbol{E}^{T} \circledast\left(\boldsymbol{C} \odot \delta_{g}\right) .
$$

\section{Fault Fuzzy Production Rule Sets}

To obtain the causality between a fault and the statuses of its protective devices, in this subsection we present fault fuzzy production rule sets for main sections including transmission lines $(L)$, buses $(B)$ and transformers $(T)$ in transmission networks.

The fault fuzzy production rules consist of propositions and certainty factors. A certainty factor represents the degree of confidence that a fault occurs. Each rule has one certainty factor. Due to the uncertainty of the knowledge of experts and senior dispatchers, we use linguistic terms to describe certainty factors.

In the sequel, we introduce the fault fuzzy production rule sets for three main sections: lines, buses and transformers.

A line has six types of protections: sending end main protections, sending end first backup protections, sending end second backup protections, receiving end main protections, receiving end first backup protections and receiving end second backup protections. The fault fuzzy production rule set for lines contains nine rules, in which the meaning of each proposition is shown in Table II:

$$
\begin{aligned}
& R_{1}\left(c_{1}=A H\right):\left(p_{1} \text { and } p_{2} \text { operate }\right) \\
& \text { ( })\left(C B_{1} \text { and } C B_{2} \text { trip }\right) \rightarrow L \text { fails } \\
& R_{2}\left(c_{2}=A H\right):\left(p_{1} \text { and } p_{4} \text { operate }\right) \\
& \text { ( }\left(C B_{1} \text { and } C B_{4} \text { trip }\right) \rightarrow L \text { fails } \\
& R_{5}\left(c_{3}=A H\right):\left(p_{1} \text { and } p_{6} \text { operate }\right) \\
& \text { (\) }\left(C B_{1} \text { and } C B_{6} \text { trip }\right) \rightarrow L \text { fails } \\
& R_{3}\left(c_{4}=A H\right):\left(p_{3} \text { and } p_{2} \text { operate }\right) \\
& \text { (1) }\left(C B_{3} \text { and } C B_{2} \text { trip } \rightarrow L\right. \text { fails } \\
& R_{4}\left(c_{5}=A H\right):\left(p_{3} \text { and } p_{4} \text { operate }\right) \\
& \text { (D) }\left(\mathrm{CB}_{3} \text { and } \mathrm{CB}_{4} \text { trip }\right) \rightarrow L \text { fails } \\
& R_{7}\left(c_{6}=A H\right):\left(p_{3} \text { and } p_{6} \text { operate }\right) \\
& \left(C \mathrm{CB}_{3} \text { and } C B_{6} \text { trip }\right) \rightarrow L \text { fails } \\
& R_{6}\left(c_{7}=A H\right):\left(p_{5} \text { and } p_{2} \text { operate }\right) \\
& \left(\text { ( }\left(\mathrm{CB}_{5} \text { and } C B_{2} \text { trip }\right) \rightarrow L\right. \text { fails } \\
& R_{8}\left(c_{8}=A H\right):\left(p_{5} \text { and } p_{4} \text { operate }\right) \\
& \text { ( })\left(\mathrm{CB}_{5} \text { and } \mathrm{CB}_{4} \text { trip }\right) \rightarrow L \text { fails } \\
& R_{9}\left(c_{9}=V H\right):\left(p_{5} \text { and } p_{6} \text { operate }\right) \\
& (1)\left(C B_{5} \text { and } C B_{6} \text { trip }\right) \rightarrow L \text { fails. }
\end{aligned}
$$

The fault fuzzy production rule set for buses contains two rules, in which the meaning for each proposition is shown in 
TABLE II

Meaning of Each Proposition in Rule Set of L

\begin{tabular}{|c|c|l|l|}
\hline$P_{1}$ & sending end main protections of $\mathrm{L}$ & $C B_{1}$ & $C B_{s}$ related to $P_{1}$ \\
\hline$P_{2}$ & receiving end main protections of $\mathrm{L}$ & $C B_{2}$ & $C B_{s}$ related to $P_{2}$ \\
\hline$P_{3}$ & sending end first backup protections of $\mathrm{L}$ & $C B_{3}$ & $C B_{s}$ related to $P_{3}$ \\
\hline$P_{4}$ & receiving end first backup protections of $\mathrm{L}$ & $C B_{4}$ & $C B_{s}$ related to $P_{4}$ \\
\hline$P_{5}$ & sending end second backup protections of $\mathrm{L}$ & $C B_{5}$ & $C B_{s}$ related to $P_{5}$ \\
\hline$P_{6}$ & receiving end second backup protections of $\mathrm{L}$ & $C B_{6}$ & $C B_{s}$ related to $P_{6}$ \\
\hline
\end{tabular}

TABLE III

Meaning of Each Proposition in Rule Set of B

\begin{tabular}{|c|c|c|c|}
\hline$P_{1}$ & main protections of $\mathrm{B}$ & $C B_{1}$ & $C B_{s}$ related to $P_{1}$ \\
\hline$P_{2}$ & backup protections of $\mathrm{B}$ & $C B_{2}$ & $C B_{s}$ related to $P_{2}$ \\
\hline
\end{tabular}

TABLE IV

MeAning of EAch Proposition in Rule Set of T

\begin{tabular}{|c|c|c|c|}
\hline$P_{1}$ & main protections of $\mathrm{T}$ & $C B_{1}$ & $C B_{s}$ related to $P_{1}$ \\
\hline$P_{2}$ & first backup protections of $\mathrm{T}$ & $C B_{2}$ & $C B_{s}$ related to $P_{2}$ \\
\hline$P_{3}$ & second backup protections of $\mathrm{T}$ & $C B_{3}$ & $C B_{s}$ related to $P_{3}$ \\
\hline
\end{tabular}

TABLE V

Confidence LeVels of the Operated Protective Devices

\begin{tabular}{|c|cc|cc|cc|}
\hline \multirow{3}{*}{ Sections } & \multicolumn{6}{|c|}{ Protective devices } \\
\cline { 2 - 7 } & \multicolumn{2}{|c|}{ Main } & \multicolumn{2}{|c|}{ First backup } & \multicolumn{2}{c|}{ Second backup } \\
\cline { 2 - 7 } & Relays & CBs & Relays & CBs & Relays & CBs \\
\hline$L$ & VH & VH & H & H & MH & MH \\
$B$ & VH & VH & - & - & MH & MH \\
$T$ & VH & VH & H & H & MH & MH \\
\hline
\end{tabular}

Table III:

$$
\begin{aligned}
& R_{1}\left(c_{1}=A H\right):\left(p_{1} \text { operates }\right) \otimes\left(\text { all or partial } C B_{1} \text { trips }\right) \\
& \quad \rightarrow B \text { fails } \\
& R_{2}\left(c_{2}=V H\right):\left(p_{2} \text { operates }\right) \otimes\left(C B_{2} \text { trips }\right) \rightarrow B \text { fails } .
\end{aligned}
$$

The fault fuzzy production rule set for transformers contains three rules, in which the meaning for each proposition is shown in Table IV:

$$
\begin{aligned}
& \quad R_{1}\left(c_{1}=A H\right):\left(p_{1} \text { operates }\right) \oplus\left(\text { all or partial } C B_{1} \text { trips }\right) \\
& \quad \rightarrow T \text { fails } \\
& R_{2}\left(c_{2}=A H\right):\left(p_{2} \text { operates }\right) \oplus\left(\text { all or partial } C B_{2} \text { trips }\right) \\
& \quad \rightarrow T \text { fails } \\
& R_{3}\left(c_{3}=V H\right):\left(p_{3} \text { operates }\right) \oplus\left(C B_{3} \text { trips }\right) \rightarrow T \text { fails. }
\end{aligned}
$$

The information obtained from the SCADA system may include operation failure, maloperation and misinformation; it is necessary to use a confidence level to describe the operation accuracy of each section. Thus, we assign an empirical confidence level to each protective device including the protective relay of each line, bus or transformer, or each of its corresponding CBs. Tables V and VI show the confidence levels of the operated protective devices and the non-operate protective devices, respectively.
TABLE VI

Confidence LeVels of the Non-Operate Protective Devices

\begin{tabular}{|c|cc|cc|cc|}
\hline \multirow{3}{*}{ Sections } & \multicolumn{6}{|c|}{ Protective devices } \\
\cline { 2 - 7 } & \multicolumn{2}{|c|}{ Main } & \multicolumn{2}{c|}{ First backup } & \multicolumn{2}{c|}{ Second backup } \\
\cline { 2 - 7 } & Relays & CBs & Relays & CBs & Relays & CBs \\
\hline$L$ & L & L & L & L & L & L \\
$B$ & ML & L & - & - & ML & L \\
$T$ & ML & L & ML & L & L & L \\
\hline
\end{tabular}

\section{Algorithmic Elaboration of FDSNP}

This subsection summarizes the FDSNP method, whose flowchart is shown in Fig. 4, as follows.

Step 1) Read operation messages about protective relays and/or CBs in a power transmission network from the SCADA system.

Step 2) Search for outage areas. We suggest network topology analysis because it decreases the number of candidate diagnosing areas and reduce the subsequent computational workload [10]. The search process is described as follows:

i) Let the search iteration $t=1$;

ii) Construct a set $Q_{t}$ of section numbers: assign a number to each section in the power transmission network. The numbers of all sections constitute the set $Q_{t}$;

iii) Construct a subset $M_{t}$ of section numbers: put the number of a randomly chosen section from $Q_{t}$ into the subset $M_{t}$. If there is a closed $\mathrm{CB}$ connecting this chosen section, find all the closed CBs connecting it, otherwise, go to vi). Find all the other sections linking with each of the closed CBs and put their numbers from $Q_{t}$ into $M_{t}$. Continue to find the closed $\mathrm{CBs}$ and sections according to those in $M_{t}$;

iv) $t$ is increased by one;

v) Construct the set $Q_{t}$ : remove the numbers of the sections in $M_{t}$ from $Q_{t-1}$ and obtain $Q_{t}$. If $Q_{t}$ is not empty, the search process goes to (iii);

vi) Find passive networks, i.e., outage areas, from $M_{1}, M_{2}, \ldots, M_{N_{s}}$, where $N_{s}$ is the maximum number of all numbers referring to section subsets. The search process stops.

Step 3) If there is only one section in the passive networks found in Step 2, this section is the faulty one and the algorithm stops, otherwise, a fault diagnosis model based on an FRSN P system is built for each section. The model-building process is described as follows. A section in the passive network is chosen randomly. According to the relay protections of the section, we design fault fuzzy production rules and then determine proposition and rule neurons and create their linking relationship to obtain the FRSN $\mathrm{P}$ system. The certainty factor of each rule is empirically set. According to Tables V and VI, we set confidence levels for main protections, first backup pro- 
TABLE VII

Status Information About Protective Relays AND CBs

\begin{tabular}{|c|c|c|}
\hline \multirow{2}{*}{ Cases } & \multicolumn{2}{|c|}{ Status information } \\
\cline { 2 - 3 } & Operated relays & Tripped CBs \\
\hline \multirow{2}{*}{1} & $B_{1 m}, L_{2 R s}, L_{4 R s}$ & $C B_{4}, C B_{5}, C B_{7}$ \\
& & $C B_{9}, C B_{12}, C B_{27}$ \\
\hline \multirow{2}{*}{$2^{*}$} & $L_{2 R s}, L_{4 R s}$ & $C B_{4}, C B_{5}, C B_{7}$ \\
& & $C B_{9}, C B_{12}, C B_{27}$ \\
\hline \multirow{2}{*}{3} & $B_{1 m}, L_{1 S p}, L_{1 R m}$ & $C B_{4}, C B_{5}, C B_{6}$ \\
& $B_{1 m}, L_{1 S m}, L_{1 R p}$ & $C B_{7}, C B_{9}, C B_{11}$ \\
\hline \multirow{3}{*}{4} & $B_{2 m}, L_{2 S p}, L_{2 R m}$ & $C B_{4}, C B_{5}, C B_{6}, C B_{8}, C B_{9}$ \\
& & $C B_{10}, C B_{11}, C B_{12}$ \\
\hline 5 & $T_{3 p}, L_{7 S p}, L_{7 R p}$ & $C B_{14}, C B_{16}, C B_{29}, C B_{39}$ \\
\hline \multirow{3}{*}{6} & $L_{1 S m}, L_{1 R p}, L_{2 S p}$ & $C B_{7}, C B_{8}, C B_{11}$ \\
& $L_{2 R p}, L_{7 S p}, L_{7 R m}$ & $C B_{12}, C B_{29}, C B_{30}$ \\
& $L_{8 S m}, L_{8 R m}$ & $C B_{39}, C B_{40}$ \\
\hline \multirow{2}{*}{$7^{*}$} & $T_{7 m}, T_{8 P}, B_{7 m}$ & $C B_{19}, C B_{20}, C B_{29}, C B_{30}$ \\
& $B_{8 m}, L_{5 S m}, L_{5 R p}$ & $C B_{32}, C B_{33}, C B_{34}, C B_{35}$ \\
& $L_{6 S s}, L_{7 S p}, L_{7 R m}, L_{8 S s}$ & $C B_{36}, C B_{37}, C B_{39}$ \\
\hline
\end{tabular}

tections, second backup protections and their CBs. Then a one-to-one relationship between the fuzzy truth value of each input neuron and the confidence level of each protection is established to obtain the initial values of the model.

Step 4) The algebraic fuzzy reasoning algorithm is used to acquire the fault confidence level of each section.

Step 5) If the confidence level $\theta$ of a section satisfies the condition $\theta \geq(0.58,0.63,0.80,0.86)$, the section is faulty, otherwise, if $\theta$ satisfies the condition $\theta$ $\leq(0.17,0.22,0.36,0.42)$, the section is not faulty, otherwise, the section may be faulty.

\section{CASe Studies}

In this section, seven cases of the local system in an EPS shown in Fig. 3 are considered as examples to test the effectiveness and superiority of FDSNP. These cases include single and multiple fault situations. The status information (with/without incompleteness and uncertainty) about protective relays and CBs is shown in Table VII, where "*" means that this case includes incomplete or uncertain status information from the SCADA system.

FDSNP is used to diagnose faults for the seven cases, and the diagnosis results are shown in Table VIII, which contains the faulty sections and their fault confidence levels. Table VIII lists the fault confidence levels of only faulty sections. In fact, FDSNP provides fault confidence levels of all diagnosed sections. Four diagnosis methods, fuzzy logic (FL) [3], fuzzy Petri nets (FPN) [4], genetic algorithm-tabu search (GATS) [15], and genetic algorithm (GA) [17], are used as benchmarks to perform comparative experiments. The reason that we choose the four methods is that they considered the same local electrical power system as FDSNP as well as the same cases. The diagnosis
TABLE VIII

Fault Sections and Their Fault Confidence LeVels OBTAINED BY USING FDSNP

\begin{tabular}{|c|c|c|c|}
\hline \multirow{3}{*}{ Cases } & \multicolumn{3}{|c|}{ Diagnosis results of FDSNP } \\
\cline { 2 - 4 } & \multirow{2}{*}{1} & \multicolumn{2}{|c|}{ Fault confidence levels } \\
\cline { 2 - 4 } & & Trapezoidal fuzzy numbers & Linguistic terms \\
\hline 2 & $B_{1}$ & $(0.975,0.98,1,1)$ & $\mathrm{VH}$ \\
\hline \multirow{2}{*}{3} & $B_{1}$ & $(0.5655,0.6174,0.80,0.86)$ & {$[\mathrm{M}, \mathrm{MH}]$} \\
\hline \multirow{4}{*}{4} & $B_{1}$ & $(0.975,0.98,1,1)$ & $\mathrm{VH}$ \\
& $L_{1}$ & $(0.9506,0.9604,1,1)$ & {$[\mathrm{H}, \mathrm{VH}]$} \\
\hline \multirow{3}{*}{5} & $B_{1}$ & $(0.975,0.98,1,1)$ & $\mathrm{VH}$ \\
& $B_{2}$ & $(0.975,0.98,1,1)$ & $\mathrm{VH}$ \\
& $L_{1}$ & $(0.9506,0.9604,1,1)$ & {$[\mathrm{H}, \mathrm{VH}]$} \\
& $L_{2}$ & $(0.9506,0.9604,1,1)$ & {$[\mathrm{H}, \mathrm{VH}]$} \\
\hline \multirow{4}{*}{6} & $T_{3}$ & $(0.72,0.78,0.92,0.97)$ & $\mathrm{H}$ \\
& $L_{7}$ & $(0.9506,0.9604,1,1)$ & {$[\mathrm{H}, \mathrm{VH}]$} \\
\hline & $L_{1}$ & $(0.702,0.7644,0.92,0.97)$ & {$[\mathrm{H}, \mathrm{VH}]$} \\
& $L_{2}$ & $(0.702,0.7644,0.92,0.97)$ & {$[\mathrm{H}, \mathrm{VH}]$} \\
& $L_{7}$ & $(0.702,0.7644,0.92,0.97)$ & {$[\mathrm{H}, \mathrm{VH}]$} \\
& $L_{8}$ & $(0.9506,0.9604,1,1)$ & {$[\mathrm{H}, \mathrm{VH}]$} \\
\hline \multirow{5}{*}{7} & $L_{5}$ & $(0.702,0.7644,0.92,0.97)$ & {$[\mathrm{H}, \mathrm{VH}]$} \\
& $L_{7}$ & $(0.702,0.7644,0.92,0.97)$ & {$[\mathrm{H}, \mathrm{VH}]$} \\
& $B_{7}$ & $(0.975,0.98,1,1)$ & {$[\mathrm{H}, \mathrm{VH}]$} \\
& $B_{8}$ & $(0.975,0.98,1,1)$ & {$[\mathrm{H}, \mathrm{VH}]$} \\
& $T_{7}$ & $(0.975,0.98,1,1)$ & {$[\mathrm{H}, \mathrm{VH}]$} \\
& $T_{8}$ & $(0.72,0.78,0.92,0.97)$ & $\mathrm{H}$ \\
\hline
\end{tabular}

results of the five methods are shown in Table IX, where "-" means that this case was not considered in the corresponding reference. It is worth pointing out that FL in [3] used only the information about CBs and the diagnosis results of GA in [17] may contain multiple solutions, such as Cases 5-7.

From Table VIII, we can see that the fault confidence levels represented by trapezoidal fuzzy numbers provide a quantitative description for the faulty sections which makes these results more reliable. The linguistic terms corresponding to these trapezoidal fuzzy numbers provide a more intuitive and flexible way for experts and dispatchers than probability values, since their knowledge usually contain linguistic terms with a certain degree of uncertainty.

From Table IX, we can see that the diagnosis results of FDSNP, in Case 1 and Cases 3-6, are the same as those in [3] and [4], in other words, FDSNP is effective in fault diagnosis of power transmission networks in power systems. Table IX also shows that, in some cases, FDSNP is superior to FL, FPN, and GA on correctly identifying fault sections. For instance, in Case 7 , the fault diagnosis result of FDSNP is different from those in [3], [4], and [17]. In this case, for section $L_{8}$, only its second backup protective relay $S_{L 8 S s}$ operated and actually, $S_{L 8 S s}$ operated as the second backup protective relay of section $B_{8}$. So in fact, $L_{8}$ is not a faulty section. For section $B_{7}$, its main protective relay $B_{7 m}$ operated and tripped its corresponding CBs, $C B_{33}, C B_{34}$, and $C B_{35}$. So $B_{7}$ is a faulty section. Thus, for Case 7, the diagnosis result of FDSNP and GATS is better than those in [3], [4], and [17]. In Cases 5-7, comparisons of diagnosis results between FDSNP and the methods in [17] 
TABLE IX

COMPARISONS BETWEEN FDSNP AND Four FAUlt Diagnosis METHODS

\begin{tabular}{|c|c|c|c|c|c|}
\hline \multirow{2}{*}{ Cases } & \multicolumn{5}{|c|}{ Diagnosis results } \\
\hline & FDSNP & FL [3] & FPN [4] & GATS [15] & GA [17] \\
\hline 1 & $B_{1}$ & $B_{1}$ & $B_{1}$ & - & $B_{1}$ \\
\hline 2 & $B_{1}$ & - & - & - & - \\
\hline \multirow{2}{*}{3} & $B_{1}$ & $B_{1}$ & $B_{1}$ & & $B_{1}$ \\
\hline & $L_{1}$ & $L_{1}$ & $L_{1}$ & - & $L_{1}$ \\
\hline \multirow{4}{*}{4} & $B_{1}$ & $B_{1}$ & $B_{1}$ & & $B_{1}$ \\
\hline & $B_{2}$ & $B_{2}$ & $B_{2}$ & - & $B_{2}$ \\
\hline & $L_{1}$ & $L_{1}$ & $L_{1}$ & & $L_{1}$ \\
\hline & $L_{2}$ & $L_{2}$ & $L_{2}$ & & $L_{2}$ \\
\hline \multirow{4}{*}{5} & & & & & (1) $T_{3}, L_{7}$ \\
\hline & $T_{3}$ & $T_{3}$ & $T_{3}$ & $T_{3}$ & (2) $T_{3}$ \\
\hline & $L_{7}$ & $L_{7}$ & $L_{7}$ & $L_{7}$ & (3) $\quad L_{7}$ \\
\hline & & & & & (4) $\mathrm{No}$ \\
\hline \multirow{4}{*}{6} & $L_{1}$ & $L_{1}$ & $L_{1}$ & $L_{1}$ & (1) $L_{1}, L_{2}$ \\
\hline & $L_{2}$ & $L_{2}$ & $L_{2}$ & $L_{2}$ & $L_{7}, L_{8}$ \\
\hline & $L_{7}$ & $L_{7}$ & $L_{7}$ & $L_{7}$ & (2) $L_{1}, L_{7}$ \\
\hline & $L_{8}$ & $L_{8}$ & $L_{8}$ & $L_{8}$ & $L_{8}$ \\
\hline \multirow{7}{*}{7} & $L_{5}$ & $L_{5}$ & $L_{5}$ & $L_{5}$ & \\
\hline & $L_{7}$ & $L_{7}$ & $L_{7}$ & $L_{7}$ & (1) $L_{5}, L_{7}$ \\
\hline & $B_{7}$ & $B_{8}$ & $B_{7}$ & $B_{7}$ & $B_{7}, B_{8}$ \\
\hline & $B_{8}$ & $T_{7}$ & $B_{8}$ & $B_{8}$ & $T_{7}, T_{8}$ \\
\hline & $T_{7}$ & $T_{8}$ & $T_{7}$ & $T_{7}$ & $(2) L_{5}, L_{7}$ \\
\hline & $T_{8}$ & & $T_{8}$ & $T_{8}$ & $T_{7}, B_{8}$ \\
\hline & & & $L_{8}$ & & \\
\hline
\end{tabular}

show that FDSNP can solve the nonuniqueness problem of the diagnosis solution, which proves the correctness of FDSNP in diagnosing faulty sections. Besides, the diagnosis results in Cases 2 and 7 show that FDSNP can obtain satisfying results in the situations with incomplete or uncertain alarm information. Therefore, from the seven typical cases, FDSNP is effective with a good accuracy in fault diagnosis of power transmission networks.

In what follows, we use Case 1 and Case 2 as examples to illustrate the steps of FDSNP.

Case 1: complete information is obtained from the SCADA system. Operated relays: $B_{1 m}, L_{2 R s}$, and $L_{4 R s}$. Tripped CBs: $C B_{4}, C B_{5}, C B_{7}, C B_{9}, C B_{12}$, and $C B_{27}$.

The search process of outage areas is described as follows.

1) Construct the set $Q_{1}$ of section numbers: $Q_{1}=$ $\{01,02,03,04,1,2,, 3,4,5,6,7,8,9,10,11,12,13,14,15$, $16,17,18,19,20,21,22,23,24,25,26,27,28\}$, where numbers $01 \sim 04$ represent four joint nodes, which are considered as active nodes, between the local power system in Fig. 3 and other parts of the power system.

2) Construct the subset $M_{1}$ of section numbers: add number 1 into $M_{1}$ and find all the closed CBs, i.e., $C B_{1}, C B_{2}$, and $C B_{3}$, connecting the section 1 . Find all the other sections,

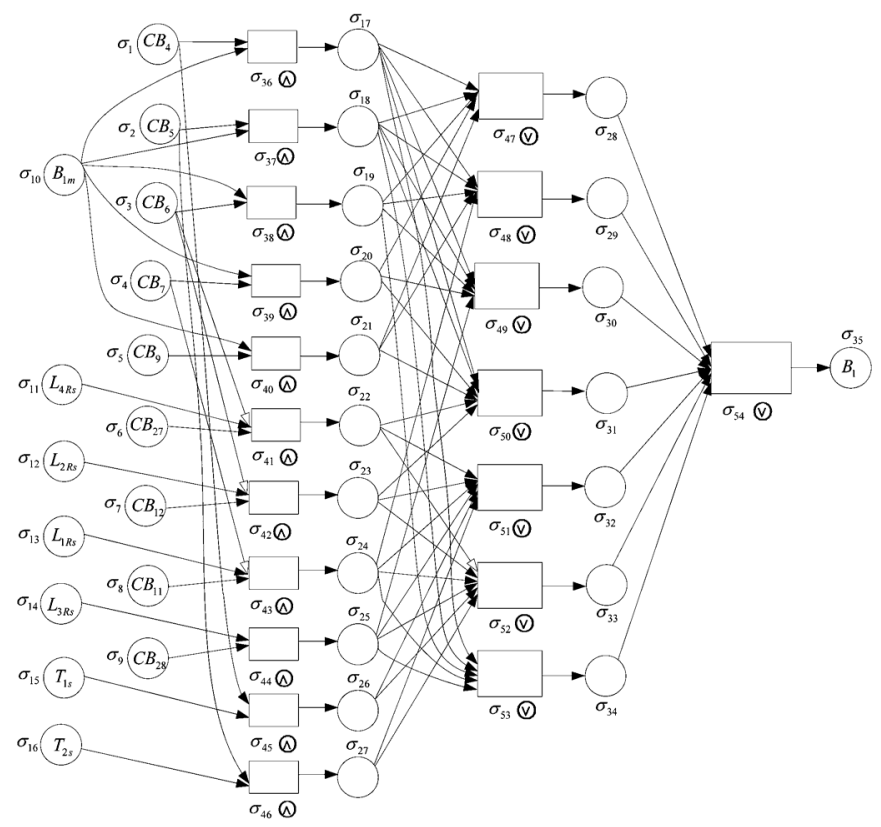

Fig. 12. Fault diagnosis model of bus $B_{1}$ based on an FRSN P system.

i.e., 01,13 , and 14 , linking with $C B_{1}, C B_{2}$, and $C B_{3}$ and add them into $M_{1}$. No other closed CB is found according to the sections in $M_{1}$. Thus, $M_{1}=\{01,1,13,14\}$.

3) Construct the set $Q_{2}$ : remove the numbers of the sections in $M_{1}$ from $Q_{1}$ and obtain $Q_{2}=\{02$ , 03, 04, 2, 3, 4, 5, 6, 7, 8, 9, 10,11,12,15, 16, 17, 18, 19, $20,21,22,23,24,25,26,27,28\}$.

4) Construct the subset $M_{2}$ : add number 2 into $M_{2}$ and execute the step 3) in Step 2 in Section III-D. We get $M_{2}=$ $\{2,3,16,20\}$.

5) Construct the set $Q_{3}$ : remove the numbers of the sections in $M_{2}$ from $Q_{2}$ and obtain $Q_{3}=$ $\{02,03,04,4,5,6,7,8,9,10,11,12,15,17,18,19,21,22$, $23,24,25,26,27,28\}$.

6) Construct the subset $M_{3}$ : add number 4 into $M_{3}$ and execute step (iii) in Step 2 in Section III-D. We get $M_{3}=$ $\{02,03,04,4,5,6,7,8,9,10,11,12,15,17,18,19,21,22$, $23,24,25,26,27,28\}$.

7) Construct the set $Q_{4}$ : remove the numbers of the sections in $M_{3}$ from $Q_{3}$ and obtain $Q_{4}=\emptyset$.

8) Find one passive network $M_{2}=\{2,3,16,20\}$ in $M_{1}, M_{2}, M_{3}$, where the numbers 2, 3, 16, 20 represent bus $B_{1}$, bus $B_{2}$, line $L_{2}$ and line $L_{4}$, respectively. The search process stops.

In what follows we take bus $B_{1}$ as an example to show how to use Steps 3-5 in Section III-D. The fault diagnosis model of $B_{1}$ based on an FRSN P system is constructed and shown in Fig. 12, where there are 35 proposition neurons and 19 rule neurons. In this figure, there are four assistant arcs (synapses), i.e., $(3,41)$, $(3,42),(4,43)$, and $(22,52)$, marked by hollow tips. We take the arc, from $\sigma_{3}$ to $\sigma_{41}$, as an example to illustrate the meaning of these assistant arcs. The arc $(3,41)$ means that if $C B_{6}$ opens, the operation of $L_{4 R s}$ and $C B_{27}$ is invalid and then the values of $L_{4 R s}$ and $C B_{27}$ are set as $(0,0,0,0)$; otherwise, the operation 
of them is valid. The fuzzy reasoning process is described as follows.

According to the alarm information in Case 1 and Tables I, $\mathrm{V}$, and VI, we obtain the trapezoidal fuzzy numbers $\boldsymbol{\theta}_{\mathbf{0}}$ and $\boldsymbol{\delta}_{\mathbf{0}}$. In our case studies, numbers $\boldsymbol{\theta}$ are vectors of dimension 35 and numbers $\boldsymbol{\delta}$ are vectors of dimension 19:

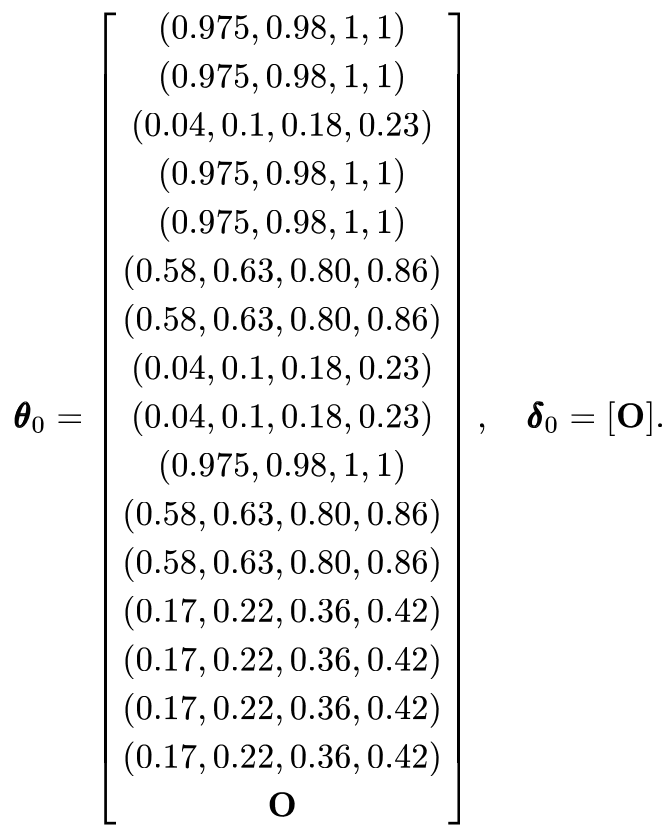

When $g=1$, we get the results

$$
\boldsymbol{\delta}_{1}=\left[\begin{array}{c}
(0.975,0.98,1,1) \\
(0.975,0.98,1,1) \\
(0.04,0.1,0.18,0.23) \\
(0.975,0.98,1,1) \\
(0.975,0.98,1,1) \\
(0.58,0.63,0.80,0.86) \\
(0.58,0.63,0.80,0.86) \\
(0.04,0.1,0.18,0.23) \\
(0.04,0.1,0.18,0.23) \\
(0.17,0.22,0.36,0.42) \\
(0.17,0.22,0.36,0.42) \\
\mathbf{O} \\
\mathbf{O} \\
\boldsymbol{\theta}_{1}=\left[\begin{array}{c} 
\\
(0.975,0.98,1,1) \\
(0.975,0.98,1,1) \\
(0.04,0.1,0.18,0.23) \\
(0.975,0.98,1,1) \\
(0.975,0.98,1,1) \\
(0.5655,0.6174,0.80,0.86) \\
(0.039,0.098,0.18,0.23) \\
(0.039,0.098,0.18,0.23) \\
(0.0156,0.2156,0.36,0.42) \\
(0.0156,0.2156,0.36,0.42) \\
\mathbf{O}
\end{array}\right] .
\end{array}\right.
$$

When $g=2$, we get the results

$$
\begin{gathered}
\boldsymbol{\delta}_{2}=\left[\begin{array}{c}
\mathbf{O} \\
(0.975,0.98,1,1)_{j=12} \\
(0.975,0.98,1,1) \\
(0.975,0.98,1,1) \\
(0.975,0.98,1,1) \\
(0.5655,0.6174,0.80,0.86) \\
(0.5655,0.6174,0.80,0.86) \\
(0.975,0.98,1,1) \\
(0,0,0,0) \\
\mathbf{O}
\end{array}\right], \\
\boldsymbol{\theta}_{2}=\left[\begin{array}{c}
(0.975,0.98,1,1)_{i=28} \\
(0.975,0.98,1,1) \\
(0.975,0.98,1,1) \\
(0.975,0.98,1,1) \\
(0.5514,0.6051,0.80,0.86) \\
(0.5514,0.6051,0.80,0.86) \\
(0.975,0.98,1,1) \\
(0,0,0,0)
\end{array}\right] .
\end{gathered}
$$

When $g=3$, we get the results.

$$
\boldsymbol{\delta}_{3}=\left[\begin{array}{c}
\mathbf{O} \\
(0.975,0.98,1,1)
\end{array}\right], \quad \boldsymbol{\theta}_{3}=\left[\begin{array}{c}
\mathbf{O} \\
(0.975,0.98,1,1)
\end{array}\right] .
$$

When $g=4$, we get the results

$$
\boldsymbol{\delta}_{4}=[\mathbf{O}] .
$$

Thus, the termination condition is satisfied and the reasoning process ends. We obtain the reasoning results, i.e., the fuzzy truth values $(0.975,0.98,1,1)$ from output neuron $\sigma_{35}$.

According to the condition in Step 5 in Section III-D, $B_{1}$ is a faulty section with a confidence level $A H$.

Similarly, we obtain that $B_{2}, L_{2}$ and $L_{4}$ have the same confidence level $(0.04,0.1,0.18,0.23)$. According to the condition in Step 5 in Section III-D, $B_{2}$, line $L_{2}$, and line $L_{4}$ are not faulty sections.

Case 2: incomplete information is obtained from the SCADA system. Operated relays: $L_{2 R s}$ and $L_{4 R s}$. Tripped CBs: $C B_{4}$, $C B_{5}, C B_{7}, C B_{9}, C B_{12}$, and $C B_{27}$. In other words, the status information about $B_{1 m}$ is missing in this case.

We use the same method as in case 1 and obtain one passive network $\{2,3,16,20\}$. In what follows we also take bus $B_{1}$ as an example to show how to use Steps 3-5 in Section III-D.

According to the alarm information in Case 2 and Tables I, $\mathrm{V}$, VI, we obtain the trapezoidal fuzzy numbers $\boldsymbol{\theta}_{\mathbf{0}}$ and $\boldsymbol{\delta}_{\mathbf{0}}$ :

$$
\boldsymbol{\theta}_{0}=\left[\begin{array}{c}
(0.975,0.98,1,1) \\
(0.975,0.98,1,1) \\
(0.04,0.1,0.18,0.23) \\
(0.975,0.98,1,1) \\
(0.975,0.98,1,1) \\
(0.58,0.63,0.80,0.86) \\
(0.58,0.63,0.80,0.86) \\
(0.04,0.1,0.18,0.23) \\
(0.04,0.1,0.18,0.23) \\
(0.17,0.22,0.36,0.42) \\
(0.58,0.63,0.80,0.86) \\
(0.58,0.63,0.80,0.86) \\
(0.17,0.22,0.36,0.42) \\
(0.17,0.22,0.36,0.42) \\
(0.17,0.22,0.36,0.42) \\
(0.17,0.22,0.36,0.42) \\
\mathbf{O}
\end{array}\right], \quad \boldsymbol{\delta}_{0}=[\mathbf{O}] .
$$


Due to limited space, only the final result of Case 2 is given. We obtain the reasoning results, i.e., the fuzzy truth values $(0.5655,0.6174,0.80,0.86)$ of the output neuron $\sigma_{35}$.

According to the condition in Step 5 in Section III-D, $B_{1}$ may be a faulty section with a confidence level $(0.5655,0.6174$, $0.80,0.86)$. Similarly, we obtain that $B_{2}, L_{2}$, and $L_{4}$ have the same confidence level $(0.04,0.1,0.18,0.23)$. According to the condition in Step 5 in Section III-D, bus $B_{2}$, line $L_{2}$, and line $L_{4}$ are not faulty sections. Therefore, we get a result that $B_{1}$ is the faulty section with a confidence level $(0.5655,0.6174,0.80$, 0.86) $(M \leq(0.5655,0.6174,0.80,0.86) \leq M H)$. Although Case 2 has incomplete alarm information, the diagnostic result is the same as that in Case 1 .

Since the analysis and fuzzy reasoning processes of other cases are similar to Cases 1 and 2, we omit them here.

\section{CONCLusions}

In this study, a graphic modeling approach, FDSNP, based on FRSN P systems with trapezoidal fuzzy numbers is presented for fault diagnosis of power transmission networks. This approach provides a good accuracy of diagnosis solutions and a rather understandable fault diagnosis process because of its intuitive illustration of graphical models and understandability of diagnosis model-building process. In addition, FDSNP can handle incomplete and uncertain messages from a SCADA system by using trapezoidal fuzzy numbers and fuzzy production rules.

Furthermore, this method can be used for large-scale power transmission networks because the complexity of the fault diagnosis models based on FRSN P systems does not increase sharply and quickly with the scale of networks. When a fault occurs, outage areas are firstly identified according to SCADA data and then fault diagnosis models for suspicious sections in each outage area are constructed [10], [15]. Thus, FDSNP avoids the construction of a large diagnosis model for an entire power transmission network by sequentially and simply constructing one for each suspicious section. This makes the computation of matrix dimensions in this fuzzy reasoning algorithm for each used fault diagnosis model is independent of the scale of the entire network. In other words, in FDSNP the complexity of the FRSN P systems used does not have a direct relationship with the scale of the network, and therefore, the complexity of the FRSN P systems does not increase sharply with the scale of the power transmission network, so it can be used in a large system.

This study proposes FDSNP and tests its validity and feasibility in diagnosing faults in power transmission networks. In this method, the set of linguistic terms and their corresponding trapezoidal fuzzy numbers are decided in an empirical way. So the following work about the choice of the set of linguistic terms and their corresponding trapezoidal fuzzy numbers, FDSNP based on software, P-Lingua [33] and MeCoSim [34], in JAVA language is now in progress. Future work will focus on verifying the performance superiority of FDSNP, compared with other diagnosis methods, by using performance metrics including diagnosis time, fault section misinformation rate, fault section missing rate and computational complexity.

\section{ACKNOWLEDGMENT}

The authors would like to thank the Editor-in-Chief, Prof. A. Conejo, the editors, and the anonymous reviewers for their insightful comments and suggestions which greatly helped in improving the quality of this paper. The authors also wish to express special gratitude to M. García-Quismondo for polishing this paper and $\mathrm{T}$. Zang for useful discussions in preparing this paper.

\section{REFERENCES}

[1] H. J. Lee, B. S. Ahn, and Y. M. Park, “A fault diagnosis expert system for distribution substations," IEEE Trans. Power Del., vol. 15, no. 1, pp. 92-97, Jan. 2000.

[2] D. Y. Ma, Y. C. Liang, X. S. Zhao, R. C. Guan, and X. H. Shi, "Multi-BP expert system for fault diagnosis of power system," Eng. Appl. Artif. Intell., vol. 26, no. 3, pp. 937-944, Mar. 2013.

[3] C. S. Chang, J. M. Chen, D. Srinivasan, F. S. Wen, and A. C. Liew, "Fuzzy logic approach in power system fault section identification," IEE Proc. Gener. Transm. Distrib., vol. 144, no. 5, pp. 406-414, Sep. 1997.

[4] J. Sun, S. Y. Qin, and Y. H. Song, "Fault diagnosis of electric power systems based on fuzzy Petri nets," IEEE Trans. Power Syst., vol. 19, no. 4, pp. 2053-2059, Nov. 2004

[5] X. Luo and M. Kezunovic, "Implementing fuzzy reasoning Petri-nets for fault section estimation," IEEE Trans. Power Syst., vol. 23, no. 2, pp. 676-685, May 2008.

[6] W. H. Chen, "Fault section estimation using fuzzy matrix-based reasoning methods," IEEE Trans. Power Del., vol. 26, no. 1, pp. 205-213, Jan. 2011.

[7] W. H. Chen, "Online fault diagnosis for power transmission networks using fuzzy digraph models," IEEE Trans. Power Del., vol. 27, no. 2, pp. 688-698, Apr. 2012.

[8] D. Thukaram, H. P. Khincha, and H. P. Vijaynarasimha, "Artificial neural network and support vector machine approach for locating faults in radial distribution systems," IEEE Trans. Power Del., vol. 20, no. 2, pp. 710-721, Apr. 2005.

[9] G. Cardoso, J. G. Rolim, and H. H. Zurn, "Identifying the primary fault section after contingencies in bulk power systems," IEEE Trans. Power Del., vol. 23, no. 3, pp. 1335-1342, Jul. 2008.

[10] J. W. Yang, Z. Y. He, and T. L. Zang, "Power system fault-diagnosis method based on directional weighted fuzzy Petri nets," Proc. CSEE, vol. 30 , no. 34 , pp. 42-49, 2010.

[11] C. F. Chien, S. L. Chen, and Y. S. Lin, "Using Bayesian network for fault location on distribution feeder," IEEE Trans. Power Del., vol. 17, no. 3, pp. 785-793, Jul. 2002.

[12] Y. L. Zhu, L. M. Huo, and J. L. Liu, "Bayesian networks based approach for power systems fault diagnosis," IEEE Trans. Power Del., vol. 21, no. 2, pp. 634-639, Apr. 2006.

[13] J. A. Hossack, J. Menal, S. D. J. McArthur, and J. R. McDonald, "A multiagent architecture for protection engineering diagnostic assistance," IEEE Trans. Power Syst., vol. 18, no. 2, pp. 639-647, May 2003

[14] E. M. Davidson, S. D. J. McArthur, J. R. McDonald, and I. Watt, "Applying multi-agent system technology in practice: Automated management and analysis of SCADA and digital fault recorder data," IEEE Trans. Power Syst., vol. 21, no. 2, pp. 559-567, May 2006.

[15] X. N. Lin, S. H. Ke, Z. T. Li, H. L. Weng, and X. H. Han, "A fault diagnosis method of power systems based on improved objective function and genetic algorithm-tabu search," IEEE Trans. Power Del., vol. 25, no. 3, pp. 1268-1274, Jul. 2010.

[16] Z. Y. He, H. D. Chiang, C. W. Li, and Q. F. Zeng, "Fault-section estimation in power systems based on improved optimization model and binary particle swarm optimization," in Proc. IEEE PES General Meeting, Canada, 2009, pp. 1-8.

[17] F. S. Wen and Z. X. Han, "Fault section estimation in power systems using a genetic algorithm," Electr. Power Syst. Res., vol. 34, no. 3, pp. 165-172, 1995.

[18] W. H. Chen, S. H. Tsai, and H. I. Lin, "Fault section estimation for power networks using logic cause-effect models," IEEE Trans. Power Del., vol. 26, no. 2, pp. 963-971, Apr. 2011.

[19] L. Tang, H. B. Sun, B. M. Zhang, and F. Gao, "Online fault diagnosis for power system based on information theory," Proc. CSEE, vol. 23, no. 7, pp. 5-11, 2003. 
[20] S. Lin, Z. Y. He, and Q. Q. Qian, "Review and development on fault diagnosis in power grid," Power Syst. Protect. Control, vol. 38, no. 4, pp. $140-150$, Feb. 2010.

[21] G. J. Xiong, D. Y. Shi, L. Zhu, and X. Z. Duan, "A new approach to fault diagnosis of power systems using fuzzy reasoning spiking neural P systems," Math. Probl. Eng., vol. 2013, p. 13, 2013, Article ID 815352.

[22] G. Păun, "Computing with membranes," J. Comput. Syst. Sci., vol. 61, no. 1 , pp. 108-143, 2000.

[23] M. Ionescu, G. Păun, and T. Yokomori, "Spiking neural P systems," Fund. Inf., vol. 71, no. 2-3, pp. 279-308, 2006.

[24] G. Păun, M. J. Pérez-Jiménez, and G. Rozenberg, "Spike train in spiking neural P systems," Int. J. Found. Comput. Sci., vol. 17, no. 4, pp. 975-1002, 2006.

[25] L. Q. Pan and G. Păun, "Spiking neural P systems: An improved normal form," Theor. Comput. Sci., vol. 411, no. 6, pp. 906-918, 2010.

[26] T. Wang, J. Wang, H. Peng, and H. Wang, "Knowledge representation and reasoning based on FRSN P systems," in Proc. WCICA, China, 2011, pp. 255-259.

[27] T. Wang, J. Wang, H. Peng, and Y. Deng, "Knowledge representation using fuzzy spiking neural P systems," in Proc. BIC-TA, China, 2010 , pp. 586-590.

[28] H. Peng, J. Wang, M. J. Pérez-Jiménez, H. Wang, J. Shao, and T. Wang, "Fuzzy reasoning spiking neural P system for fault diagnosis," Inf. Sci., vol. 235, pp. 106-116, Jun. 2013

[29] J. Wang, P. Shi, H. Peng, M. J. Pérez-Jiménez, and T. Wang, "Weighted fuzzy spiking neural P system," IEEE Trans. Fuzzy Syst., vol. 21, no. 2, pp. 209-220, Apr. 2013.

[30] G. X. Zhang, H. N. Rong, F. Neri, and M. J. Pérez-Jiménez, “An optimization spiking neural $P$ system for approximately solving combinatorial optimization problems," Int. J. Neural Syst., vol. 24, no. 5, pp. $1-16,2014$.

[31] M. Prais and A. Bose, "A topology processor that tracks network modifications over time," IEEE Trans. Power Syst., vol. 3, no. 3, pp. 992-998, Aug. 1988.

[32] S. M. Chen, "A fuzzy reasoning approach for rule-based systems based on fuzzy logics," IEEE Trans. Syst., Man, Cybern. B, vol. 26, no. 5, pp. 769-778, Oct. 1996.

[33] Research Group on Natural Computing, University of Seville: The P-Lingua [Online]. Available: http://www.p-lingua.org

[34] Research Group on Natural Computing, University of Seville: The MeCoSim [Online]. Available: http://www.p-lingua.org/mecosim

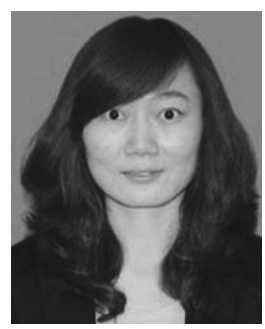

Tao Wang is pursuing the Ph.D. degree in electrical engineering at the Southwest Jiaotong University, Chengdu, China.

Now she is a visiting student at the Department of Computer Science and Artificial Intelligence, University of Sevilla, Sevilla, Spain. Her research interests include membrane computing, electric power system fault diagnosis, and soft computing.

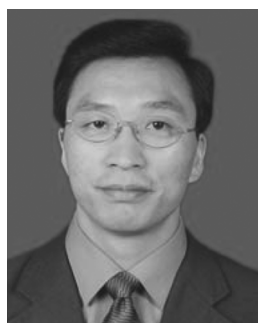

Gexiang Zhang (M'03) received the B.Sc. and M.Sc degrees from Southwest University of Science and Technology, Mianyang, China, in 1997 and 2002, respectively, and the Ph.D. degree from Southwest Jiaotong University, Chengdu, China, in 2005.

Since 2005, he has been a Professor at the School of Electrical Engineering in Southwest Jiaotong University, where he leads the research group of NatureInspired Computation and Smart Grid (NICSG). His research interests include natural computing, such as membrane computing, evolutionary computation and DNA computing, bioinformatics, and smart grid. He has published over 100 scientific papers in international journals or conferences.

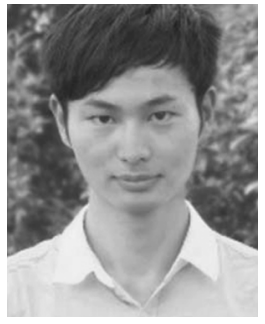

Junbo Zhao (S'13) is pursuing the Ph.D. degree at Southwest Jiaotong University, Chengdu, China.

His research interests include power system state estimation, power system operation and control, signal processing, optimization methods and their applications in robot control, and smart grid.

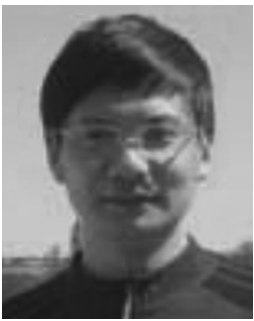

Zhengyou He (M'09-SM'13) was born in Sichuan Province, China, in 1970. He received the B.Sc. and M.Sc. degrees from Chongqing University, Chongqing, China, in 1992 and 1995, respectively, and the Ph.D. degree from Southwest Jiaotong University, Chengdu, China, in 2001.

Since February 2002, he has been a Professor at the Department of Electrical Engineering in Southwest Jiaotong University. His research interests are in the area of signal process and information theory and its application in electrical power system, and application of wavelet transforms in power system.

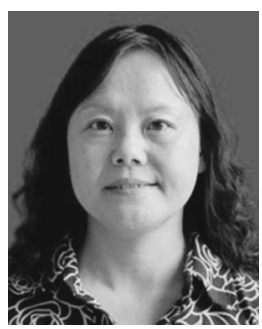

Jun Wang received the B.Sc. degree and the M.E degree in industry automation from Chongqing University, China, in 1988 and 1991, respectively, and the Ph.D. degree in electrical engineering from the Southwest Jiaotong University, China, in 2006.

She was a lecturer in the Sichuan College of Science and Technology, China (1991-2003). She has been a Professor in the Electrical and Information Engineering Department at the Xihua University since 2004. Her research field is electrical automation, intelligent control, and membrane computing.

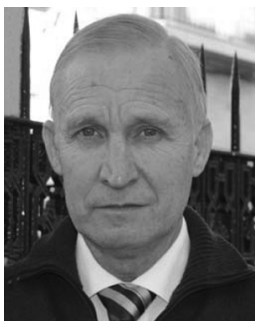

Mario J. Pérez-Jiménez received the degree in mathematics from the Barcelona University, Barcelona, Spain, in 1971, and the Ph.D. degree in mathematics from the University of Sevilla, Sevilla Spain, in 1992.

Currently, he is a numerary member of the Academia Europaea (The Academy of Europe), and a full Professor in computer science and artificial intelligence, University of Sevilla, where he is the head of the Research Group on Natural Computing. From 2005 to 2007, he was a Guest Professor of the Huazhong University of Science and Technology, Wuhan, China. His main research interests include theory of computation, computational complexity theory, natural computing (DNA computing and membrane computing), bioinformatics and computational modeling for systems biology, and population dynamics. He has published thirteen books in computer science and mathematics, and over 250 scientific papers in international journals (collaborating with researchers worldwide).

Prof. Pérez-Jiménez is a member of the editorial board of four ISI journals: Fundamenta Informaticae, International Journal of Computers Communications and Control, Natural Computing, and Theoretical Computer Science. He has been the first scientific awarded with "Important Contributions to Membrane Computing" under the auspices of the European Molecular Computing Consortium, Edinburgh, 2008. He is the main researcher in various European, Spanish, and Andalusian research grants. He has been an independent expert to the evaluation of NEST (New and Emergent Science and Technology) proposals under the Sixth Framework Programme of the European Community, and since May 2006 he has been a European Science Foundation peer reviewer. 